\title{
Annular lesion of the lung in sarcoidosis
}

\author{
HIROSHI TADA, RYOJI YASUMIZU, SHIRO YUASA, MASASHI SATO, \\ YASUO MIZUNO
}

\author{
From the Section of Internal Medicine, Himeji Red Cross Hospital, Tatsuno-cho, Himeji, Japan
}

ABSTRACT A 20 year old man presented with eight thin walled, ring shaped shadows, $1-3 \mathrm{~cm}$ in diameter, on a chest radiograph. Pulmonary sarcoidosis was diagnosed on the basis of histopathological examination of a cervical lymph node, transbronchial lung biopsy, and increased activity of angiotensin converting enzyme and lysozyme in serum. The lesions disappeared within six months of his starting corticosteroid treatment.

Various pulmonary manifestations of sarcoidosis have been documented,' including cavitary and infiltrating shadows on the chest radiograph. Nine patients with cavity like annular shadows have been reported, ${ }^{2-6}$ but the patients had only a few lesions and responded poorly to corticosteroid treatment. We present a patient with eight ring shadows, four of which disappeared completely with corticosteroid treatment.

\section{Case report}

A 20 year old man was admitted for evaluation of an abnormal chest radiograph. He had a history of cigarette smoking (one pack a day), and had been well except for occasional cough and sputum. He denied haemoptysis, fever, and night sweats, but had noticed a $3 \mathrm{~kg}$ weight loss over one year. Lymph node swelling in the cervical and axillary regions was found. The tuberculin skin test response was negative, and sputum examination showed no acid fast bacilli, fungi, or neoplastic cells. Angiotensin converting enzyme and lysozyme activities in serum were raised. Pulmonary function was normal. Pathological examination of the right cervical lymph node and transbronchial lung biopsy specimen showed multiple confluent epithelioid granulomas with giant cells and no caseous necrosis. A diagnosis of sarcoidosis was made.

The chest radiograph showed right paratracheal and bilateral hilar lymphadenopathy, fine granular shadows in both lower zones, several patchy infiltrates, and thin walled ring shaped shadows (fig 1). Subsequent radiological examinations showed seven annular shadows $(1-3 \mathrm{~cm}$ in diameter) and 15 patchy shadows $(0 \cdot 5-3 \mathrm{~cm})$. The chest tomogram and computed tomogram showed the ring shadows as circular in three dimensions (fig 2). A tomogram taken two months later showed an eighth ring shadow at the

Address for reprint requests: Dr Hiroshi Tada, Section of Internal Medicine, Himeji Red Cross Hospital, Tatsuno-cho, Himeji, Japan.

Accepted 9 June 1989 exact location of the one of the previous patchy shadows. The other ring shadows enlarged slowly. Corticosteroid treatment (prednisone $30 \mathrm{mg} /$ day) was followed by substantial clearance of the parenchymal infiltrations, disappearance of the hilar adenopathy, and a decrease in size and disappearance of the ring shadows within two months. Within six months of his starting corticosteroid treatment the thickness of the ring walls had decreased, though three had not changed their internal size appreciably and remained as small bullae. Four had diminished in size and disappeared. The largest one decreased in size but remained as a faint radio-opaque shadow.

\section{Discussion}

Cavitary lesions are rarely found in sarcoidosis, other than bullae, ectatic bronchi, and lesions secondary to infection. Nine cases have been published, ${ }^{2-6}$ seven in men and two in women (aged 20-35 years). In these cases the diagnosis of sarcoidosis was made by excluding mycobacterial or fungal infection and neoplasm, and by the presence of bilateral hilar

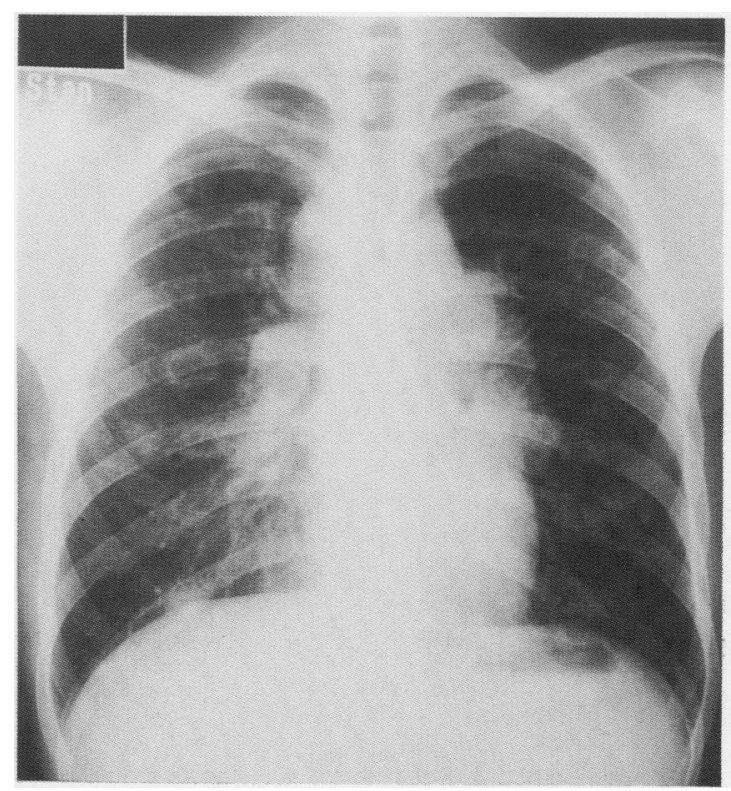

Fig 1 Chest radiograph at the first visit showing bilateral hilar lymphadenopathy, fine granular infiltrate, patchy infiltrates, and seven ring shadows. 


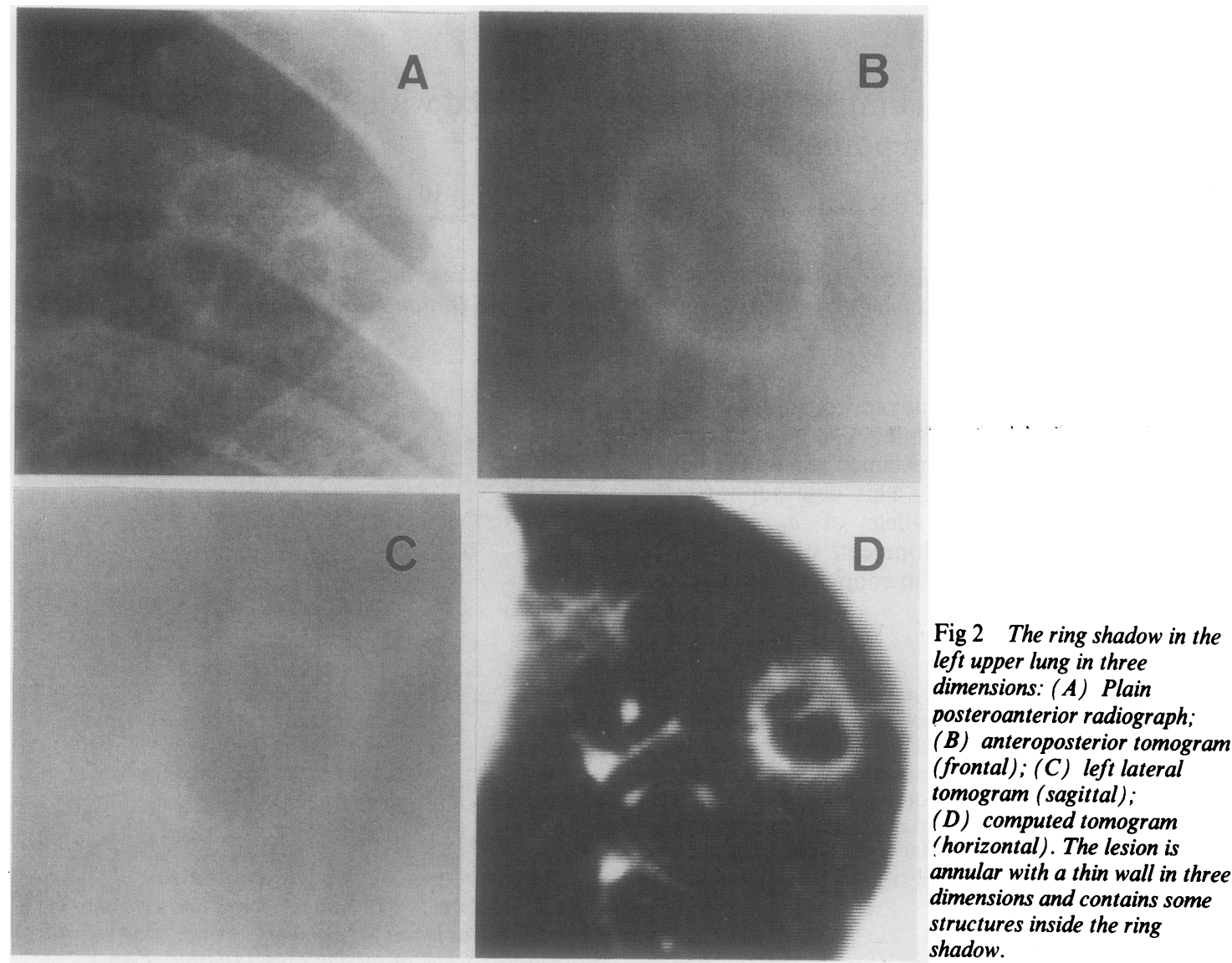

adenopathy in seven cases. The number of cavitary lesions was one in five cases, two in two cases, and three in two cases. The cavitary lesions were observed at the sites of previous patchy shadows in five cases, similar to the eighth lesion of our case. Four patients (with nine ring shadows) were treated with corticosteroids; three of the ring shadows disappeared.

Several mechanisms have been proposed to explain the formation of the ring shaped, cavity like lesion, a finding sometimes $^{2-4}$ but not always ${ }^{36}$ supported by histopathological examination. These include ischaemic necrosis of a mass of hyaline fibrosis, coalescence of granulomas, and air trapping secondary to sarcoid granuloma formed in bronchial mucosa. Three shadows in our patient, as in the shadows reported in published cases, appeared to be cavitary according to the tomograms and computed tomograms. The other five shadows, one of which is shown in figure 2, may not be cavities for the following reasons: (1) the lesions appear to contain something inside the cavity (fig 2); (2) they had a regular, spherical shape as if they were artefacts; (3) their walls were very thin; and (4) they disappeared rapidly in response to corticosteroid.

Sarcoidosis is well documented as a disease of immunological pathogenesis with compartmentalised and immunological dysregulation. ${ }^{7}$ We have assumed that a similar mechanism might cause the annular erythematous or granulomatous lesions seen occasionally in the skin in sarcoidosis and in other immunological disorders. A similar lesion in the lung in three dimensions would result in thin walled spherical lesions. A reaction of inflammatory or granulomatous type (or both) might diffuse through the spongy structure of the lung, causing an orange peel like lesion to be formed at the front line of the reaction. The findings on the chest radiograph and responsiveness to corticosteroid in our patient fit this possibility well, as the annular skin lesions respond well to corticosteroid treatment. The exact mechanism, however, will not be clear until more precise histological and immunopathological investigations have been performed.

\section{References}

1 Freundlich IM, Libshitz HI, Glassman LM. Sarcoidosis: typical and atypical thoracic manifestations and complications. Clin Radiol 1970;21:376-83.

2 Hamilton R, Petty TL, Haiby G. Cavitary sarcoidosis of the lung. Arch Intern Med 1965;116:428-30.

3 Bistrong HW, Tenney RD, Sheffer AL. Asymptomatic cavitary sarcoidosis. JAMA 1970;213:1030-2.

4 Tellis MCJ, Putnam JS. Cavitation in large multinodular pulmonary disease. A rare manifestation of sarcoidosis. Chest 1977;71:792-3.

5 Rohatgi PK, Schwab LE. Primary acute pulmonary cavitation in sarcoidosis. AJR 1980;134:1199-203.

6 Jones DK, Dent RG, Rimmer MJ, Flower CDR. Thin-walled ring shadows in early pulmonary sarcoidosis. Clin Radiol 1984; 35:307-10.

7 Muller-Quernheim J, Saltini C, Sondermeyer P, Crystal RG. Compartmentalized activation of the interleukin 2 gene by lung $T$ lymphocytes in active pulmonary sarcoidosis. $J$ Immunol 1986;137:3475-83. 